\title{
Multiple metastases from myxoid liposarcoma (MLS) for which Whole Body MRI (WB-MRI) was useful for their detection
}

\author{
Katsuyuki Nakanishi ${ }^{*}$, Yoshinori Imura ${ }^{2}$, Shigenori Nagata ${ }^{3}$, Akio Tsukabe $^{1}$, Norifumi Naka $^{2}$, Shinichi Nakatsuka ${ }^{3}$ and Noriyuki Tomiyama ${ }^{4}$ \\ ${ }^{1}$ Department of Diagnostic \& Interventional Radiology, Osaka International Cancer Institute, Japan \\ ${ }^{2}$ Department of Orthopaedic Surgery, Osaka International Cancer Institute, Japan \\ ${ }^{3}$ Pathology and Cytology, Osaka International Cancer Institute, Japan \\ ${ }^{4}$ Department of Diagnostic \& Interventional Radiology, Osaka University Graduate School of Medicine, Japan
}

\begin{abstract}
The present case is that of a 50-year-old male with myxoid liposarcoma (MLS) in his left thigh, which metastasized to multiple unusual sites. In WB-MRI, multiple abnormal signal areas were shown in the bilateral femurs, ilium, vertebral bodies, ribs and peritoneal cavity, and these areas were judged as metastases. However, in FDG-PET, no distant metastases were proved. After finishing the chemotherapy, follow-up WB-MRI was performed and the reduction of the primary mass and all metastatic areas were proved. In all areas, apparent diffusion coefficient (ADC) values of diffusion-weighted images (DWI) were increased and chemotherapeutic effect was proved. For detecting metastases from MLS, WB-MRI including DWI was considered to be as one of the fundamental modalities. It is useful not only for detecting lesions, but also for monitoring the chemotherapeutic effect.
\end{abstract}

\section{Clinical Presentation}

\section{Background}

Liposarcoma is one of the most common soft-tissue sarcomas in adults, with peak incidence between 40 and 60 years old [1-4]. MLS with round cell components have a tendency to metastasize to unusual sites including soft-tissue, retroperitoneum, mediastinum, chest wall, lymph node and so on [1]. It is well known that MRI is a highly sensitive method to detect abnormal bone marrow [1-6]. FDG-PET measures glucose uptake into the tumor itself. These characteristics allow PET to detect small volumes of disease before a secondary cortical bone reaction appears [1]. However, it is also well known that in low grade soft-tissue sarcomas, specificity and sensitivity are limited [3-5]. WBMRI including DWI has recently been widely used in the oncologic field for detecting metastatic lesions [7-12]. This case report is that of the multiple extrapulmonary metastases of MLS of the left thigh, which were detected by the WB-MRI including DWI. Also, DWI could evaluate the chemotherapeutic effect.

\section{Case Report}

For one year, 50-year-old-male had been feeling discomfort and having a swelling in his left thigh. This swelling had enlarged gradually and he visited the orthopedic department. An approximately $10 \times 15 \mathrm{~cm}$ movable soft mass was palpable in his left thigh. There was no Tinel's sign. On CT examination, fat containing low density mass was shown in his left thigh. Considering to the large diameter, irregular margin and inhomogeneous density, malignant soft tissue tumor was highly suspected. Moreover, a density of the bone marrow of adjacent left femur seemed to be slightly high and was suspected of being either bone metastasis or direct invasion of this mass. In MRI (Figure 1), this mass was shown as low intensity on T1-weighted images (T1WI), and as high intensity on T2-weighted images (T2WI). On PET-CT examination, this mass was shown as high accumulation and its standardized uptake value (SUV) max was 2.8. However, no distant metastases were proved (Figure 2).

\section{Histopathologic examination of the biopsy specimen with molecular investigation}

Hematoxylin and eosin ( $\mathrm{H} \& \mathrm{E})$ stained glass slide revealed two patterns of histologic features: paucicellular and cellular patterns (Figure 3). The former pattern was characterized by small cells with mild nuclear atypia accompanied by myxoid stroma and arborizing vasculature, whereas the latter by cellular sheets of back-to-back uniform round-cells with a high nuclear to cytoplasmic ratio, but lacking pleomorphism, which had shown up in $20 \%$ of the whole sample. Unequivocal lipoblasts were not identified in either component. Reverse transcriptase-polymerase chain reaction (RT-PCR) using the formalin-fixed and paraffin-embedded tissues demonstrated that those samples harbored TLS-CHOP chimeric transcripts, confirming myxoid liposarcoma with $>5 \%$ round-cell component.

After that, WB-MRI examination was performed for verifying the existence of distant metastases (Figure 4). In WB-MRI, multiple abnormal signal areas were shown in the bilateral femurs, ilium,

${ }^{*}$ Correspondence to: Katsuyuki Nakanishi MD, $\mathrm{PhD}$, Department of Diagnostic \& Interventional Radiology, Osaka International Cancer Institute, 3-1-69, Otemae, Chuo-ku, Osaka 541-8567, Japan, E-mail: je2k-nkns@asahi-net.or.jp

Received: December 01, 2018; Accepted: December 04, 2018; Published: December 10,2018 

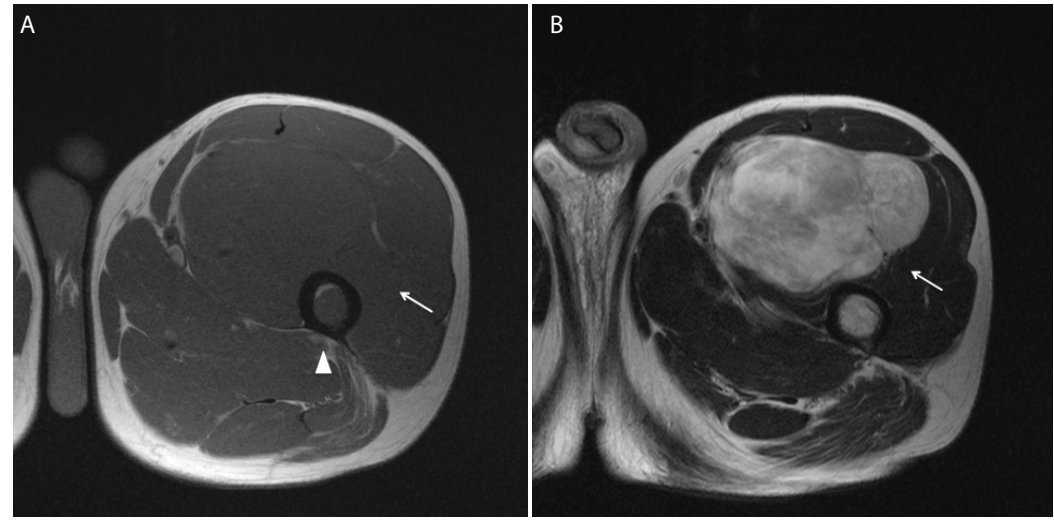

Figure 1. 50-year-old-male. For a year, he had been feeling discomfort and having a swelling in his left thigh. Initial MRI.

(a)In axial T1-weighted image, this mass is shown as iso intensity comparing with surrounding muscle (white arrow) and the bone marrow of the left femur is also shown as low intensity (white arrowhead)

(b)In axial T2-weighted image, this mass is shown as inhomogeneous high intensity (white arrow).

Through the whole slices of MRI, fat signal components are few.
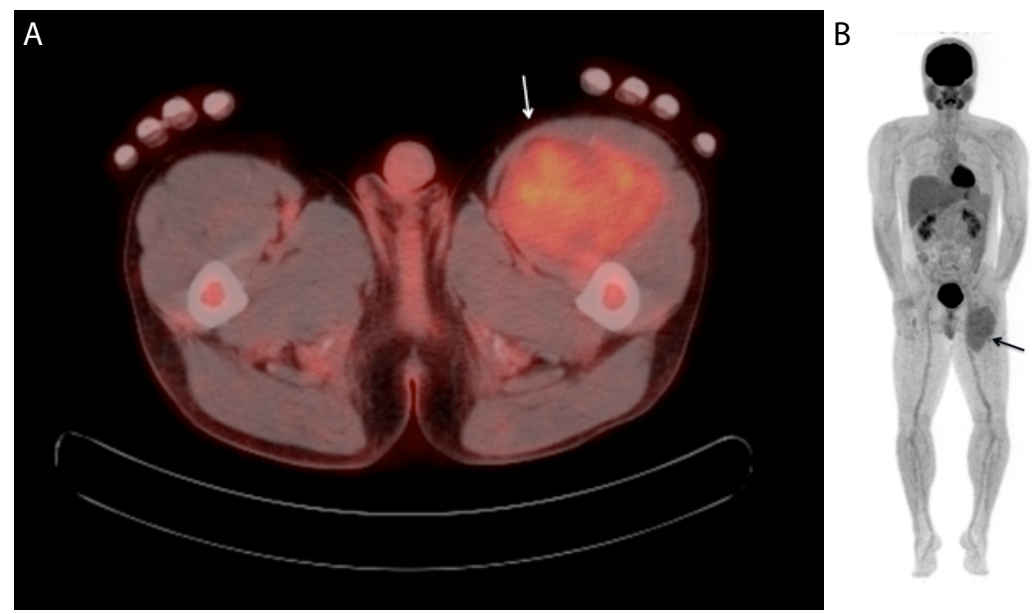

Figure 2. ${ }^{18}$ F-Fluorodeoxyglucose-positron emission tomography/CT (FDG-PET/CT)

(a) In axial CT fused image, this mass is shown as mild uptake of FDG (white arrow). Its SUV max is 2.8 and also the bone marrow of the left femur is also shown as mild uptake and its SUV max is 2.4. It was suspected of direct invasion of bone metastasis, however this is not certain.

(b) Whole body PET-MIP image shows the uptake of the mass in the left thigh (arrow). No other uptake is shown.
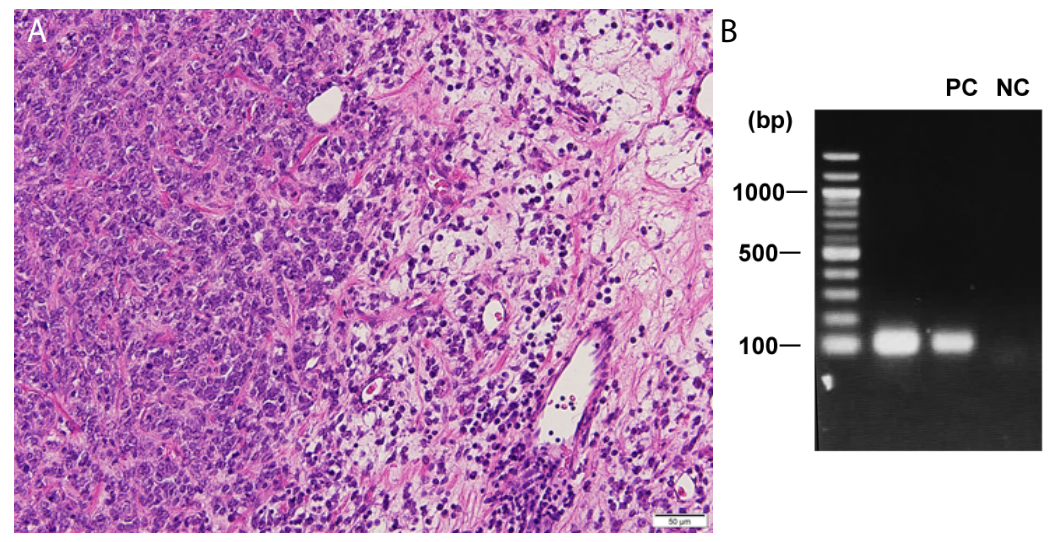

Figure 3. Histopathologic examination of the biopsy specimen with molecular investigation.

(a) Histologic findings of the biopsy specimen. Cellular (left) and paucicellular (right) patterns compatible with poorly-differentiated and well-differentiated components of myxoid liposarcoma, respectively (H \& E, x 200).

(b) Detection of fusion transcripts by RT-PCR. Lane 1 (on the left), molecular-size marker; lane 2, the present case of tumor with TLS-CHOP chimeric transcript of type 2 (110 bp); lane 3 , $\mathrm{PC}$, positive control at our institute (a previous case of myxoid liposarcoma); lane 4, NC, negative control (distilled water). 

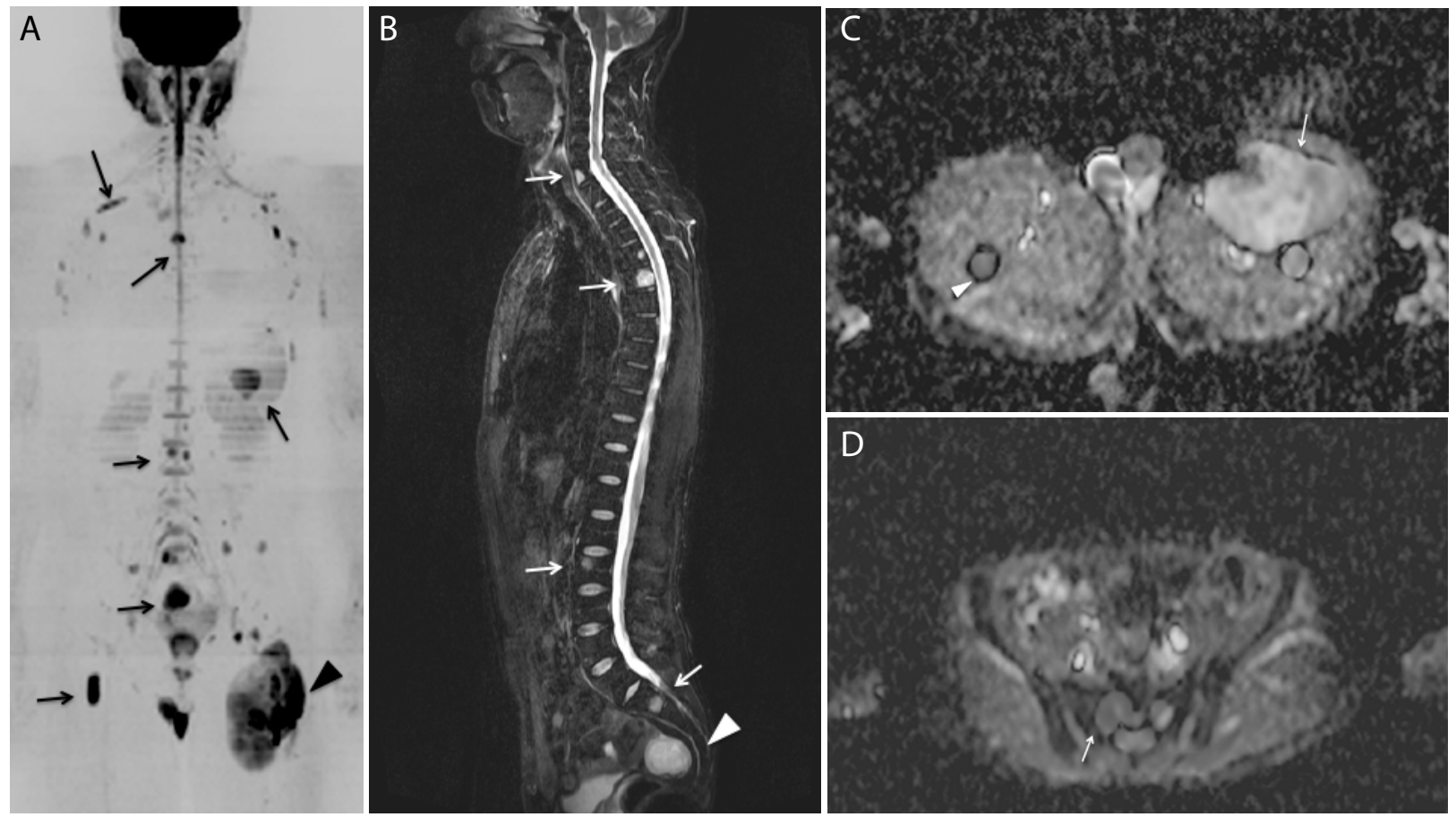

Figure.4 After proving myxoid liposarcoma histopathologically, whole body MRI was performed for checking the distant metastases.

(a)In coronal MIP image of DWI (b value $=1000 \mathrm{~s} / \mathrm{mm}^{2}$ ), the primary mass in the left proximal thigh is shown (arrowhead). Moreover, multiple high intensity areas are shown in the vertebral bodies, right proximal femur, level of pelvis rib and so on (arrows).

(b)In total spine sagittal Short-Tau Inversion Recovery (STIR) image, high intensity areas are shown in C7, Th5, L3 vertebral body (white arrows) and also high intensity area is shown in the presacral region (white arrowhead), which are also shown as high intensity in DWI (Figure 5A). They were judged as metastases.

(c)In Apparent Diffusion Coefficient (ADC) map image, the primary mass in the left proximal thigh reveals as inhomogenous high intensity and its $\mathrm{ADC}$ value is $2.0\left(\mathrm{x} 10^{-3} \mathrm{~mm} / \mathrm{sec}\right)($ white arrow). Another low intensity is shown in the left femur (white arrowhead) and its ADC value is 1.1.

(d) In ADC map, the low intensity area is shown in the sacrum (white arrow). Its ADC value is 1.0.

vertebral bodies, ribs and peritoneal cavity. These areas were judged as multiple bone metastases and dissemination of the peritoneal cavity.

This patient was treated with $1.2 \mathrm{mg} / \mathrm{m}^{2}$ of trabectedin once every 3 weeks.

After finishing three courses of chemotherapy, follow-up WBMRI revealed the reduction of the primary mass and all metastatic areas (Figure 5). In all areas, signals of high b DWI were decreased and ADC values of DWI were increased. These findings proved the chemotherapeutic effect.

\section{Discussion}

MLS is the second most common subtype of liposarcoma, and usually occurs in deep soft tissues of the extremities, arising primarily within the musculature of the thigh [1]. It has been previously observed that MLS tends to metastasize to unusual soft tissue or bone locations [1-6].

MRI has been rated as a highly sensitive technique to detect distant metastases, and the site and size of the primary tumor, which generally presents fatty septa or small adipose nodules in a myxoid mass. On the contrary, Schweb et al. described that whole-body FDG-PET had an unacceptably low sensitivity for the detection of spinal metastases [3-5]. Hence, WB-MRI is a competent tool for detecting metastatic lesions of MLS. To the best of our knowledge of past literature, there are two reports that described the usefulness of WB-MRI to detect metastatic lesions of MLS [11,12]. However, in these two reports,
DWI was not used. In oncology, DWI has emerged as a powerful tool to detect the tumoral involvement of bone, and other organs, by metastases, myeloma, or lymphoma and so on [7]. Inversion-recovery fat-suppressed DWI using high-diffusion sensitizing gradients (b values) is usually performed in the axial plane at consecutive anatomic stations [7]. The use of two or more b values enables one to combine the acquisition of diagnostic images with the calculation of ADC values. Areas of restricted diffusion in highly cellular areas show low ADC values compared with less cellular areas that return higher $\mathrm{ADC}$ values. In our department, WB-MRI examination, body DWIs are obtained from axial plain from lower neck to proximal femur with $b$ value of 0 and 1000 . Additionally, coronal reconstruction and radial maximal intensity projection (MIP) images of DWI are obtained. Paruhikunnan et al. described [13] that the reliability of WB-DWI as the sole imaging sequence for the detection of lymph nodal and pulmonary metastases is poor. We also consider that the spatial resolution of DWI is insufficient. Hence, for screening whole body, combining DWI and conventional sequences such as STIR and T1-weighted images is important. In fact, in our department, total examination time of WB-MRI including DWI and conventional sequences was approximately 23 minutes [14]. In our present case, DWI-MIP image is comprehensible for detecting various metastatic spreads and their chemotherapeutic response. However, for evaluating the signal of DWI, we have to consider the "T2 shinethrough" effect.

The signal of DWI depends on both water diffusion and the T2 relaxation time. 

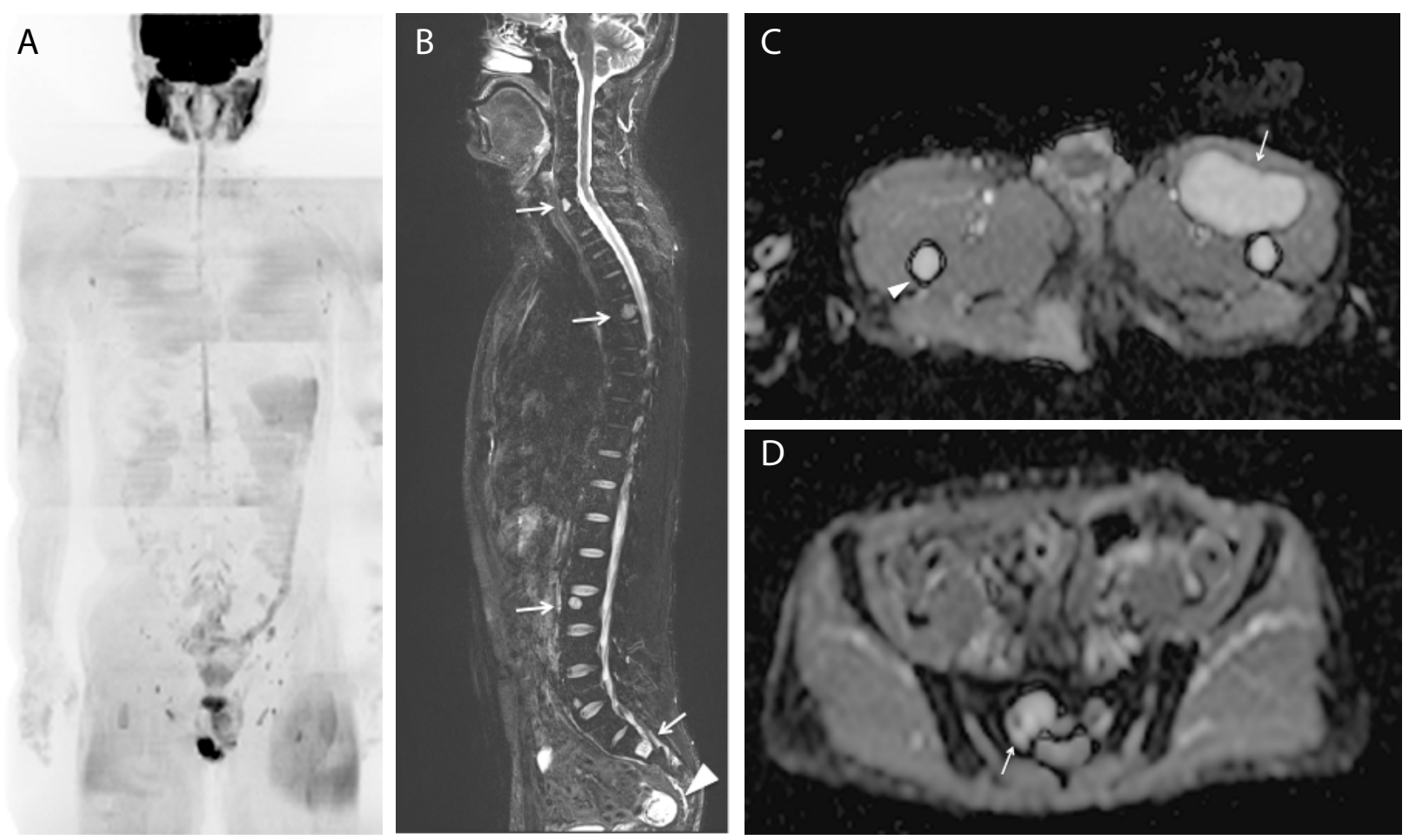

Figure 5. MRI about four months after initial examination after administration of 3 cycles of trabectedin $1.2 \mathrm{mg} / \mathrm{m}^{2}$

(a)In MIP image of DWI, multiple high intensity areas disappear comparing with pretreatment image Figure 4A.

(b)Unlike DWI, in STIR, high intensity areas in C7, Th5, L3 vertebral body (white arrows) remain. Also, the presacral mass remains but decreases its size (white arrowhead).

(c)On ADC map, the size of the primary mass in the left proximal thigh decrease and the signal increase, thus ADC value increase to 2.4 (white arrow). Also, the signal of the bone marrow of the left femur (white arrowhead) increases to 2.3 .

(d)On ADC map, the signal in the sacrum increases (white arrow). Its ADC value increases to 2.4.

In the study by Einarsdóttir et al. [9], the highest ADC values for sarcoma were found in an MLS. Nagata et al. [10] found that although there was no significant difference between benign and malignant myxoid tumors, nonmyxoid malignant tumors had a lower mean ADC $\left(0.94 \times 10^{-3} \mathrm{~mm}^{2} / \mathrm{s}\right)$ than nonmyxoid benign lesions $\left(1.31 \times 10^{-3} \mathrm{~mm}^{2} / \mathrm{s}\right)$. In our MLS case, left thigh primary site reveals as high ADC value (2.0), but metastatic sites reveal as low to intermediate ADC value (1.0-1.5). After administering Trabectedine, the primary site decreased its size and also its ADC value increased. All other metastatic sites decreased their sizes, their signal in high b DWI, but their ADC values increased. These processes reflect the good effect of chemotherapy.

\section{Conclusion}

We summarized that for detecting extrapulmonary multiple metastases from MLS, WB-MRI including DWI should be selected as one of the screening modalities. It is useful not only for detecting lesions, but also for monitoring the chemotherapeutic effect.

\section{Learning Point}

1. The metastases of MLS are well known for the difficulty detecting using FDG-PET.

2. WB-MRI including DWI might be selected as one of the screening modalities.

\section{Consent}

Written informed consent

\section{References}

1. Conill C, Setoain X, Colomo L, Palacín A, Combalia-Aleu A, et al. (2008) Diagnostic efficacy of bone scintigraphy, magnetic resonance imaging, and positron emission tomography in bone metastases of myxoid liposarcoma. J Magn Reson Imaging 27: 625-628. [Crossref]

2. Noble JL, Moskovic E, Fisher C, Judson I (2010) Imaging of skeletal metastase in myxoid liposarcoma. Sarcoma (Article ID 262361)

3. Schwab JH, Boland PJ, Antonescu C, Bilsky MH, Healey JH (2007) Spinal metastases from myxoid liposarcoma warrant screening with magnetic resonance imaging. Cancer 110: 1815-1822. [Crossref]

4. Schwab JH, Boland P, Guo T, et al. (2007) Skeletal metastases in myxoid liposarcoma: an unusual pattern of distant spread. Ann Surg Oncol 14: 1507-1514. [Crossref]

5. Sakamoto A, Fukutoku Y, Matsumoto Y, Harimaya K, Oda Y, et al. (2012) Myxoid liposarcoma with negative features on bone scan and [18F]-2-fluoro-2-deoxy-Dglucose-positron emission tomography. World J Surg Oncol 10: 214. [Crossref]

6. Ishii T, Ueda T, Myoui A, Tamai N, Hosono N, et al. (2003) Unusual skeletal metastases from myxoid liposarcoma only detectable by MR imaging. Eur Radiol 13: L18591. [Crossref]

7. Lecouvet FE (2016) Whole-Body MR Imaging: Musculoskeletal Applications. Radiology 279: 345-365. [Crossref]

8. Koh DM, Collins DJ (2007) Diffusion-weighted MRI in the body: applications and challenges in oncology. AJR Am J Roentgenol 188: 1622-1635. [Crossref]

9. Einarsdóttir H, Karlsson M, Wejde J, Bauer HC (2004) Diffusion-weighted MRI of soft tissue tumours. Eur Radiol 14: 959-963. [Crossref]

10. Nagata S, Nishimura H, Uchida M, Sakoda J, Tonan T, et al. (2008) Diffusion weighted imaging of soft tissue tumors: usefulness of the apparent diffusion coefficient for differential diagnosis. Radiat Med 26: 287-295. [Crossref] 
11. Stevenson JD, Watson JJ, Cool P, Cribb GL, Jenkins JP, et al. (2016) Whole-body magnetic resonance imaging in myxoid liposarcoma: A useful adjunct for the detection of extra-pulmonary metastatic disease. Eur J Surg Oncol 42: 574-580. [Crossref]

12. Seo SW, Kwon JW, Jang SW, Jang SP, Park YS (2011) Feasibility of whole-body MRI for detecting metastatic myxoid liposarcoma: a case series. Orthopedics 34: e748-54. [Crossref]
13. Paruthikunnan SM, Kadavigere R, Karegowda LH (2017) Accuracy of Whole-Body DWI for Metastases Screening in a Diverse Group of Malignancies: Comparison With Conventional Cross-Sectional Imaging and Nuclear Scintigraphy. AJR Am J Roentgenol 209: 477-490. [Crossref]

14. Nakanishi K, Sakai M, Sumikawa H, Kanayama N, Oshima K et al. (2017) Bone metastases from head \& neck squamous cell carcinoma (HNSCC) - Reviewing the patients' background and imaging features mainly of whole body MRI (WBMRI). Cancer Rep Rev 1: 1-5.

Copyright: (C2018 Nakanishi K. This is an open-access article distributed under the terms of the Creative Commons Attribution License, which permits unrestricted use, distribution, and reproduction in any medium, provided the original author and source are credited. 